Check for updates

Cite this: Phys. Chem. Chem. Phys. $2021,23,25830$

Received 15th June 2021 Accepted 2nd November 2021

DOI: $10.1039 / \mathrm{d} 1 \mathrm{cp} 02702 \mathrm{f}$

rsc.li/pccp

\title{
Structural determinants of a permeation barrier of the SecYEG translocon in the active state $\uparrow$
}

\author{
Ekaterina Sobakinskaya, (D) * Heinrich Krobath, (D) Thomas Renger (D) and \\ Frank Müh (D)
}

\begin{abstract}
The SecYEG translocon is a channel in bacteria, which provides a passage for secretory proteins across as well as integration of membrane proteins into the plasma membrane. The molecular mechanism, by which SecYEG manages protein transport while preventing water and ion leakage through the membrane, is still controversial. We employed molecular dynamics simulations to assess the contribution of the major structural elements - the plug and the pore ring (PR) - to the sealing of SecYEG in the active state, i.e., with a signal sequence helix occupying the lateral gate. We found, that the PR alone can provide a very tight seal for the wild-type translocon in the active state for both water and ions. Simulations of the mutant $1403 \mathrm{~N}$, in which one of the PR-defining isoleucine residues is replaced with asparagine, suggest that hydrophobic interactions within the PR and between the PR and the plug are important for maintaining a tight conformation of the wild-type channel around the PR. Disruption of these interactions results in strong fluctuations of helix TM7 and water leakage of the translocon.
\end{abstract}

\section{Introduction}

Many bacterial proteins, including most secretory proteins, are transported across the plasma membrane through a channel that is formed by a conserved membrane protein complex, called the translocon. ${ }^{1}$ This channel provides a lateral exit into the bilayer for membrane proteins, while simultaneously offering a pathway across the membrane for secretory proteins.

The bacterial translocon is a heterotrimeric complex named SecYEG, consisting of a large subunit, SecY, featuring ten transmembrane helices (TM), and the two smaller subunits SecE and SecG (Fig. 1). ${ }^{2}$ A side cut through the translocon reveals that SecY has an hourglass shape with a constriction in the center of the membrane. Important structural elements of SecY include (i) the central pore ring (PR) in the constriction region, composed of hydrophobic amino acids (Fig. 1), (ii) the lateral gate (LG) formed by transmembrane helices TM2b and TM7 (depicted in green and orange, respectively, in Fig. 1), and (iii) the partly helical re-entrant loop termed TM2a, usually called the "plug" (yellow in Fig. 1). The PR consists of six aliphatic amino acids, usually isoleucine (Ile) residues, which project their hydrophobic side chains inside the channel

Institute for Theoretical Physics, Johannes Kepler University Linz, Altenberger Strasse 69, A-4040 Linz, Austria. E-mail: ekaterina.sobakinskaya@jku.at $\dagger$ Electronic supplementary information (ESI) available: Table S1 and Fig. S1-S15, description of the independent-samples $t$-test used to analyze averaged interaction energies. See DOI: $10.1039 / \mathrm{d} 1 \mathrm{cp} 02702 \mathrm{f}$
(Fig. 1a and b). Four of them are of particular interest, since they determine the minimal radius of the PR, and, thus, the space available for a translocating substrate. They are located on helices TM5, TM10, TM2b, and TM7 (Fig. 1a). The LG is open in the beginning of the translocation and provides an exit for membrane proteins into the surrounding lipid bilayer during the next stages of translocation. It also accommodates the signal sequence (SS) of secretory proteins (Fig. 1c). The SecE unit contains an amphipathic helix and plays the role of a clamp, keeping the two halves of SecY together. SecG makes only few contacts with SecY and appears to be of minor importance for the function of the translocon.

Numerous experimental and theoretical studies explored how the channel and its interaction partners translocate proteins (see recent reviews ${ }^{1-3}$ and references therein), but a detailed molecular description of the conformational transitions of the channel during protein transport is still lacking. Such a picture is of particular importance for understanding how the translocon prevents leakage of small molecules, i.e. ions or metabolites, during translocation.

Experimental studies of the permeability of the channel's resting state demonstrated that wild type (WT) SecYEG was impermeable for ions and water. ${ }^{4,5}$ On the contrary, the plugdeletion mutant $\operatorname{Sec} \mathrm{Y}(\Delta \mathrm{P})$ and translocons with mutations in the PR were found to be leaky for chloride $\left(\mathrm{Cl}^{-}\right)$ions. ${ }^{4,5}$ However, $\operatorname{SecY}(\Delta \mathrm{P})$ was sealed for sodium $\left(\mathrm{Na}^{+}\right)$, potassium $\left(\mathrm{K}^{+}\right)$or sulfate ions $\left(\mathrm{SO}_{4}{ }^{2-}\right)$, thus, still providing a barrier to some ions. ${ }^{5}$ This effect can explain the functionality of the 


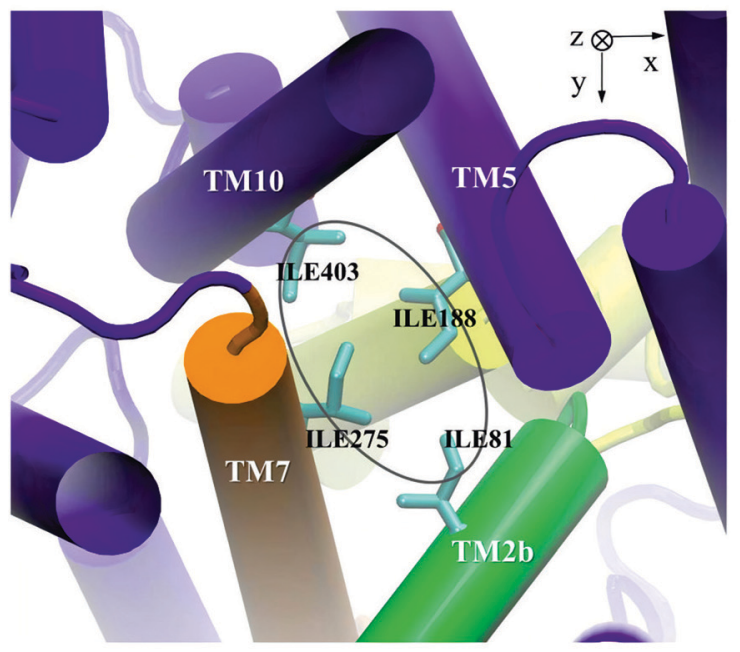

a

C

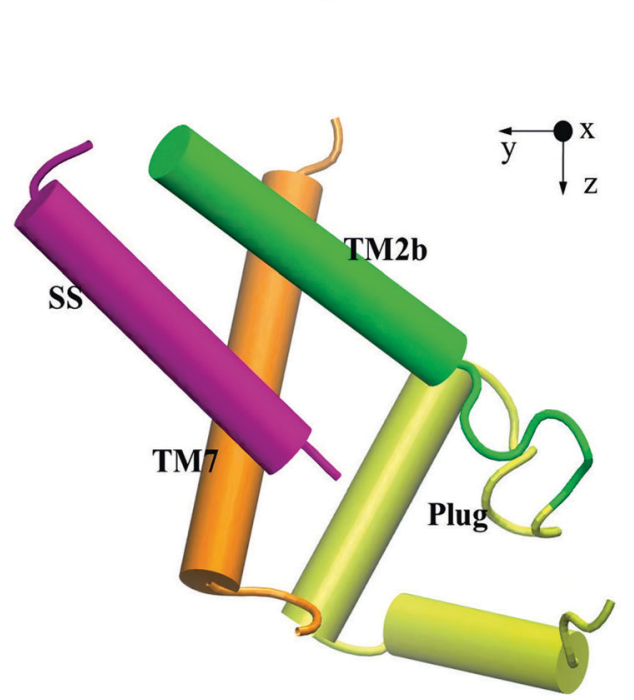

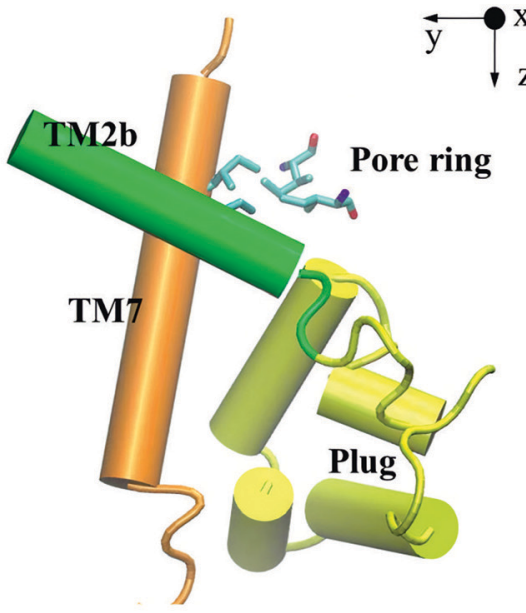

b

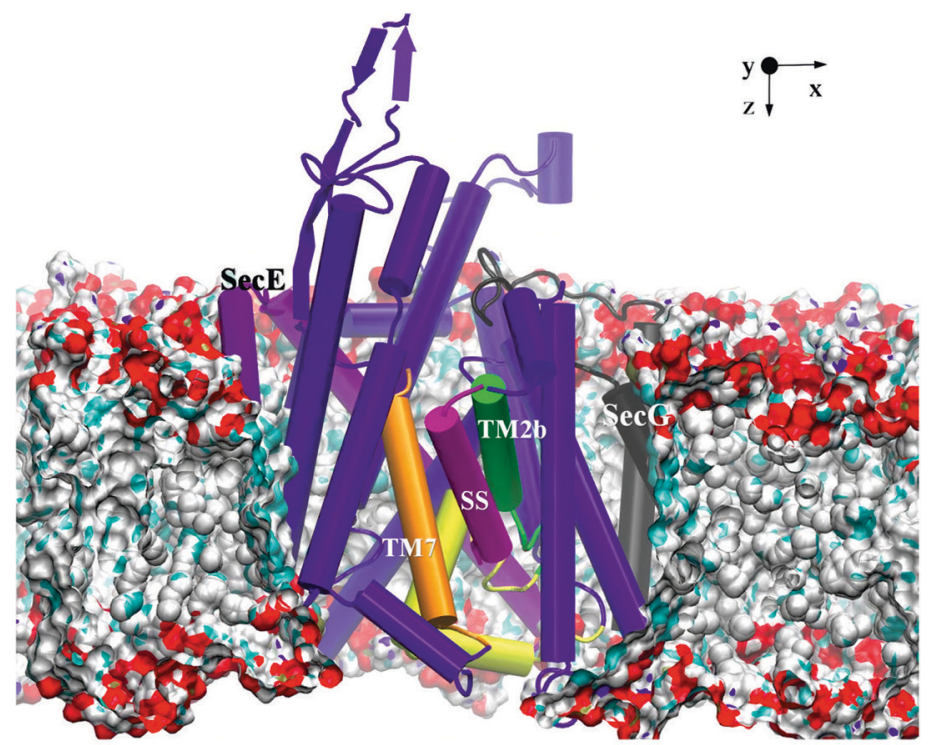

d

Fig. 1 Architecture of the SecYEG translocon: ( $a$ and b) crystal structure of the resting state, (c and d) modified structure "WT-closed". (a) Crystal structure of SecYEG from Thermus thermophilus in the resting state according to PDB ID 5AWW; view from the cytosolic side into the channel (flanked by TM2b, TM5, TM7, and TM10) onto the pore ring (PR) with residues lle 81, lle 188, lle 275, and lle 403. The ellipse indicates the widths of the PR as measured on the basis of the center-of-mass positions of the lle residues. The plug is seen in the background. (b) Same as in a, but viewed along the membrane plane (cytoplasm top) and showing besides the lle residues only TM2b, TM7, and the plug region. (c) Modified structure "WT-closed" with the signal sequence (SS) inserted into the lateral gate (LG) between TM2b and TM7 as described in the text (Section 2); viewed along the membrane plane and showing only TM2b, TM7, SS, and the plug. (d) Complete modified structure "WT-closed" inserted into the membrane (cytoplasm top). The $z$-axis is normal to the membrane plane and points towards the periplasm (see also Fig. 2).

plug-deletion mutants that was established in experiments with E. coli cells. ${ }^{6}$ In the case of the PR mutants, SecYEG became progressively leakier with increasing number of the mutations. ${ }^{5}$ Moreover, the most severe mutants caused a strong growth defect. Thus, these investigations demonstrate that both PR and plug are necessary to effectively seal the resting translocon.

At the same time, the results of molecular dynamics (MD) simulations revealed that the PR poses the dominant barrier for water flux, whereas the plug serves to suppress fluctuations of the PR. ${ }^{7}$ The importance of the interactions between plug and
PR was also confirmed in a structural analysis of the plugdeleted channel, ${ }^{6}$ revealing that a new plug can be formed. Being smaller than the original one, the new plug seemed to lose most interactions with TM2b, TM7, and PR residues, which can lead to a destabilization of the translocon's closed state.

Experimental studies of the translocon in the active state with inserted substrate showed that the WT channel is sealed for small molecules and ions. ${ }^{5,8}$ On the contrary, permeation of $\mathrm{Cl}^{-}$ions through active $\operatorname{Sec} \mathrm{Y}(\Delta \mathrm{P})$ was inhibited only moderately by short nascent chains, and reduced by about $90 \%$ by 
channel-inserted peptides. ${ }^{5}$ For the active channel with mutations in the PR, a high permeability for $\mathrm{Cl}^{-}$ions was observed regardless of the sequence of the peptide inside the translocon.

The above results indicate that the PR plays an important role in sealing the channel both in the resting and the active states. For the resting channel, though, interactions between the PR and the plug were also found to be important. Taking into account that the active state is characterized by an expanded PR and has the plug moved down, the question arises, whether the PR-plug interactions still contribute to the sealing and, if so, what kind of interactions are important.

Several studies aimed at elucidating the intercommunication between the PR and the plug, but the reported results remain controversial. MD simulations performed on the crystal structure of Methanocaldococcus jannaschii SecYEG (PDB ID $1 \mathrm{RH} 5)^{9}$ showed that expanding the PR is a more effective way to destabilize the plug than intercalation of a cylinder-like probe at the lateral gate. ${ }^{10}$ In the latter case, no considerable movement of the plug toward the external side of SecY was observed in contrast to the case, where the pore ring was expanded by passing virtual soft balls.

On the other hand, MD simulations made on a similar structure from the same organism (PDB ID 1RHZ) ${ }^{9}$ revealed that opening of the lateral gate was sufficient to break the contact between the plug and the rest of the channel, leading to plug detachment. ${ }^{11}$ An understanding of the PR interactions is further complicated by the fact that all MD simulations of the active channel were performed with "artificial" systems: either the open LG was kept fixed in a certain position like in ref. 11 and 12 or the substrate was pushed through without proper opening of the LG. ${ }^{10,13,14}$

In recent years, high resolution structures of the active channel containing a translocating peptide were reported. ${ }^{15}$ Besides, a high-resolution structure of the resting channel with a well-resolved plug was also published. ${ }^{16}$ Combining both, we prepared a natural active state of SecYEG with a signal sequence (SS) in a proper position in the LG. This conformation is characterized by an expanded PR and resembles the initial stage of translocation, when the SS is already interposed between LG helices, but the translocating substrate is not yet inserted in the PR. Since activation of the channel can be accompanied by a destabilization of the plug (see ref. 1 and references therein), we also built a setup with the plug shifted away from the PR. These conformations were used to study water leakage as well as free energy barriers for ion passage across the channel. Furthermore, we discuss the contributions of different electrostatic interactions in the system to the free energy barrier.

\section{Methods}

\subsection{Overview of setups}

The core unit of the simulation is the SecYEG channel in the resting state obtained from the Thermus thermophilus crystal structure (PDB ID 5AWW) ${ }^{16}$ with a resolution of $2.7 \AA$
(Fig. 1a and b). The structural model of the active state was created by mimicking the positions of the LG helices TM2 and TM7 of the structure with PDB ID 5EUL (Fig. 1c and d). The latter structure ${ }^{15}$ is a chimera of SecYE from Geobacillus thermodenitrificans and SecA from Bacillus subtilis containing the SS of OmpA (outer membrane protein A) and a short polypeptide following it. Thus, this structure corresponds to the open channel with a SS in the LG and a peptide loop inside the pore. Superposition of the crystal structures 5AWW and 5EUL demonstrates a drastic shift of TM7, TM8, and TM2b (ESI, $\dagger$ Fig. S1). These significant movements of LG helices represent our current understanding of how the channel is opened by insertion of the SS.

$\mathrm{NAMD}^{17}$ was used to pull helices TM7, TM8, and TM2b apart, thereby widening the LG (for details, see Section 2.2). The final configuration of our SecYEG model contains the SS (for amino acid sequence, see Section 2.2) in the LG, but not the peptide loop in the channel (Fig. 1c and d). In the following, this configuration is named "WT-closed", where "closed" refers to the closed position of the plug. To elucidate the role of the latter in sealing the channel, a setup with an open plug was created by shifting it down from the PR (for details, see Section 2.3). The resulting configuration with the SS in the LG is referred to as "WT-open".

To study the contribution of the PR to the barrier, the mutation I403N was introduced into the PR of SecYEG. This mutation corresponds to one of the amino acid changes in the prlA4 mutant ${ }^{4}$ (which, however, is a change of Ile 408 in E. coli). The mutant setups are referred to as "MUT-closed" and "MUTopen" depending on the position of the plug.

The setups WT-closed and WT-open also formed the basis for studying the transport of $\mathrm{K}^{+}$and $\mathrm{Cl}^{-}$ions through the channel. Depending on the ion type and plug position, we have prepared the following systems: "Cl-1q-open", "Cl-1q-closed" and "K-1q-open". Here, "1q" refers to the charge state of Asp 410 (see below).

Additional setups were created to study the influence of a charged residue of SecYEG on the passage of the ions. The analysis of the distribution of amino acids inside translocons from different organisms revealed the presence of charged residues in spatial proximity to the $\mathrm{PR}$ in bacteria and archaea (see ESI, $\dagger$ Table S1). These charged residues may affect the translocation process by exerting electrostatic forces. To uncover the influence of Asp 410 in T. thermophilus, we calculated the free energy profiles for $\mathrm{Cl}^{-}$and $\mathrm{K}^{+}$ions in the channel with charged ("1q" case) or uncharged ("0q" case) Asp 410. The " $0 q$ " case was only studied with open plug, resulting in the setups "Cl-0q-open" and "K-0q-open". The case of uncharged Asp 410 was approximated by setting all atomic partial charges of the side chain to zero. All other titratable groups were modelled in their standard protonation state in all setups.

\subsection{Systems assembly}

To obtain a channel conformation with open LG, we first performed an overlay of the structures 5AWW and 5EUL based on the SecE subunit, controlling also the match of the helices 
TM9. The result demonstrated that not only TM2b and TM7 are to be displaced in order to open the 5AWW channel, but also TM8, which is necessary to provide space for the shifted TM7 (ESI, $\dagger$ Fig. S1d). Finally, the SS was placed inside the LG according to the guidelines given by the 5EUL structure. The amino acid sequence of the SS construct with net charge $+2 e$ is as follows: KKTAIAIAVALAGFAT.

The assembly protocol included the following steps: (i) CHARMM-GUI ${ }^{18-20}$ was used to add missing atoms to the 5AWW structure, which subsequently was embedded in a lipid bilayer composed of 296 lipid molecules: 70\% dioleoyl-glycerophosphatidyl-ethanolamine (DOPE) and 30\% dioleoyl-glycerophosphatidyl-glycerol (DOPG) as well as a water box with dimensions $105 \times 105 \times 113 \AA$, containing 26888 water molecules as well as $100 \mathrm{mM} \mathrm{KCl}$ to mimic a bacterial membrane and physiological ion conditions. ${ }^{8,21}$ The total number of ions was $48 \mathrm{Cl}^{-}$and $123 \mathrm{~K}^{+}$to maintain charge neutrality including protein and lipids. (ii) To create an open LG, helices TM2, TM7, and TM8 were pulled by an external constant force, using steered molecular dynamics (SMD), ${ }^{22}$ to the positions of the corresponding helices in the 5EUL structure. The resulting structure of the active channel with open LG, referred to as ac5AWW, is shown overlaid with 5EUL in ESI, $\dagger$ Fig. S2. (iii) CHARMM $^{19}$ was used to place the SS in the ac5AWW structure. (iv) Minimization and equilibration of the final setup. For example, the WT-closed conformation is compared to ac5AWW in ESI, $\dagger$ Fig. S3 and to 5EUL in ESI, $\dagger$ Fig. S4. Interestingly, the LG helices in the WT-closed conformation undergo a small shift relative to their positions in ac5AWW. It is also seen that in the WT-closed setup, TM7 is tilted more towards the channel interior and the plug is closer to the PR as compared to 5EUL, since WT-closed does not contain a peptide inside the channel.

\subsection{Simulation protocols}

General information. The MD simulations were performed with $\mathrm{NAMD}^{17}$ version 2.12 along with the CHARMM36m force field. $^{20,23,24}$ The TIP3P model was used for water molecules. All simulations were done under NPT conditions: constant temperature control was regulated with Langevin dynamics ${ }^{25}$ with a $5 \mathrm{ps}^{-1}$ damping coefficient coupled to all heavy atoms $(T=300 \mathrm{~K})$; constant pressure was maintained at 1 atm by a Nosé-Hoover Langevin piston barostat ${ }^{26,27}$ with decay period 100 fs and a damping time of 50 fs. Periodic boundary conditions were applied in all dimensions. To compute electrostatic interactions, the particle-mesh Ewald summation was applied with a grid spacing of $1 \AA$, and the short-range real-space interactions were cut off at $12 \AA$ using a smooth switch function beginning at $10 \AA$. The equations of motion were integrated in 2 fs steps using the velocity Verlet algorithm. Bonded forces and short-ranged non-bonded forces were recomputed every 2 fs, long-ranged non-bonded forces every 4 fs. The SHAKE algorithm was employed to constrain the length of the bonds involving hydrogen atoms. Coordinates were saved every 4 ps. The images were prepared with VMD. ${ }^{28}$
Minimization protocol. All assembled systems were energy minimized with CHARMM ${ }^{19}$ using 500 steps of steepest descent followed by 500 steps of the Newton-Raphson algorithm.

Equilibration protocol. The energy-minimized systems were equilibrated in seven consecutive runs of 1 ns with applied restraints and an eighth run of $5 \mathrm{~ns}$ with all restraints removed. In the first run, harmonic-restraint forces with a force constant of $5 \mathrm{kcal}\left(\mathrm{mol} \AA^{2}\right)^{-1}$ were applied to the protein backbone and of $0.5 \mathrm{kcal}\left(\mathrm{mol} \AA^{2}\right)^{-1}$ to all atoms of lipids. In the next six runs, the restraints of the protein backbone were slowly released: 2,1 , $0.5,0.1,0.05,0.01 \mathrm{kcal}\left(\mathrm{mol} \AA^{2}\right)^{-1}$. The restraints for lipids were $0.2 \mathrm{kcal}\left(\mathrm{mol} \AA^{2}\right)^{-1}$ in the second run and set to 0 in the subsequent runs.

Unbiased dynamics simulation protocol. For all setups (WTclosed, WT-open, MUT-closed, MUT-open), we run 100 ns trajectories to determine the channel's water permeability. The conditions of these simulations are described in General information. Corresponding RMSD plots are given in ESI, $\dagger$ Fig. S5.

Application of external forces. External forces, provided by different protocols of NAMD, were used to (i) open the LG, (ii) pull the plug down, and (iii) monitor translocation of the ions through the pore ring.

(i) The lateral gate of the channel $5 \mathrm{AWW}$ was opened by constant-force pulling of helices TM2, TM7, and TM8, employing a TCL protocol ${ }^{29}$ of NAMD. ${ }^{17}$ The force was applied to all atoms of a pulled helix, while other atoms were kept fixed. The total time of TM2 shifting was 550 ps. Moving TM8 and TM7 to new positions required both shifts and rotations and was produced during $33 \mathrm{~ns}$ and $38 \mathrm{~ns}$ simulations, respectively. Forces were recalculated every 500 steps. The simulations were carried out under the conditions described in the General information section.

(ii) The conformation of the SecYEG channel with open plug was created by application of a constant force provided by a TCL protocol. ${ }^{29}$ The force was applied to the plug backbone atoms (residues 40 to 64), while other backbone atoms were restrained with a force constant of $1 \mathrm{kcal}\left(\mathrm{mol} \AA^{2}\right)^{-1}$ exept residues 30 to 39 and residues 65 to 75 , which were kept free. Pulling-forces were recalculated every 300 steps. The total time of plug pulling was 2 ns and $2.6 \mathrm{~ns}$ for WT and mutant, respectively. Other conditions of the simulations are described in General information.

(iii) SMD with constant velocity protocol, implemented in NAMD, was used to pull an ion through SecYEG to obtain the average force and to create windows for umbrella sampling (US). The pulling was performed with a velocity of $40 \AA \mathrm{ns}^{-1}$ and a force constant of $7 \mathrm{kcal}\left(\mathrm{mol} \AA^{2}\right)^{-1}$. To estimate the force exerted on the ion passing the channel, we ran 30 SMD trajectories of $1 \mathrm{~ns}$, which differ in starting velocities. Estimation of the force was obtained by averaging of the results over the reaction coordinate and ensemble.

Umbrella sampling. The reaction coordinate for $\mathrm{US}^{30-32}$ was chosen as the distance between the ion and the $\mathrm{C}_{\alpha}$ atom of Ile 403 in the PR (Fig. 2). The span of the reaction coordinate was subdivided into windows with an approximate $1 \AA$ spacing 


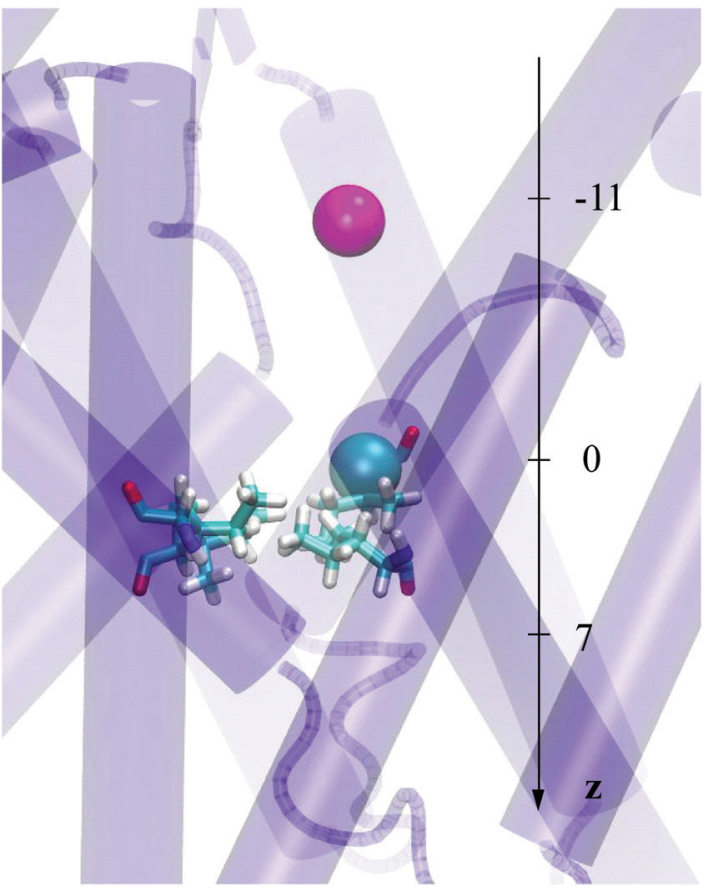

Fig. 2 Definition of the reaction coordinate for umbrella sampling of ions The $z$-coordinate of the $\mathrm{C}_{\alpha}$-atom of lle 403 (cyan sphere) defines the origin of the $z$-axis (in $\AA$ ). All lle residues forming the PR are shown in stick mode. An ion close to the position $z=-11 \AA$ is shown in magenta.

(cf. ESI, $\dagger$ Fig. S6). The fluctuations in $x$ - and $y$-direction were negligible compared to those in the restrained $z$-coordinate within the channel. For each window, an independent simulation of $6 \mathrm{~ns}$ was performed. Appropriate force constants of $2.5 \mathrm{kcal}\left(\mathrm{mol} \AA^{2}\right)^{-1}$ were selected, allowing neighboring windows to overlap significantly. We applied the Weighted Histogram Analysis Method (WHAM) $)^{32,33}$ to construct the potential of mean force (PMF) from the sorted trajectory files obtained with US. The unbiased probability distribution of a system along the reaction coordinate is calculated by a weighted average of the distributions of the individual windows. The weights for the windows are chosen in order to minimize the statistical error of the whole distribution. The Version 2.0.9 of the WHAM package was used. ${ }^{34}$ The first $1 \mathrm{~ns}$ in each window was treated as an equilibration phase and, hence, ignored for post-processing.

Computation of pairwise interactions. To calculate the difference in ion-environment interactions between the position of the ion in the channel mouth (cf. Fig. 2) and in the PR area, we created $60 \mathrm{~ns}$ trajectories corresponding to the distances between an ion and the PR of $-11 \AA$ (Traj1) and $-1 \AA$ (Traj2) for all four setups with open plug. A harmonic restraint potential with a force constant of $9.0 \mathrm{kcal}\left(\mathrm{mol} \AA^{2}\right)^{-1}$ was imposed on an ion to fix its position in the $z$-direction. The NAMD protocol for pairwise interactions ${ }^{35}$ was employed to compute electrostatic and van der Waals energies. The data set obtained from a $60 \mathrm{~ns}$ trajectory included 120 points and represented the interaction energy averaged over 500 ps. Averaging should eliminate any correlations in translational and rotational motion of water molecules between neighboring points. For the setup "K-1qopen", a 20 ns equilibration was necessary to obtain mean energy differences with a sufficient statistical significance.

Calculation of the pore ring axes (diameter). The ellipseshaped pore ring has its major axis defined between residues Ile 81 of TM2b and Ile 403 of TM10, and the minor axis is determined between residues Ile 188 of TM5 and Ile 275 of TM7 (see Fig. 1a). The axis length is calculated as the distance between the centers of mass (c.o.m.) of the corresponding residues and designated TM5-TM7 for the minor axis and TM2-TM10 for the major axis.

Water flux. As a permeation event, we counted all water molecules that crossed the position of the $z$-coordinate of the pore ring c.o.m. and moved at least $10 \AA$ beyond it. The PR c.o.m. was defined with respect to the four Ile residues forming the PR.

\section{Results}

\subsection{Water permeability of the channel}

We evaluated the SecYEG permeability by calculating the number of water molecules that crossed the channel during a (normal) MD simulation. For this purpose, we ran trajectories of 100 ns: In the setups "WT-closed", "WT-open", "MUTclosed", and "MUT-open", 11, 10, 7, and 284 water molecules, respectively, crossed the channel in both directions during this time period. (A bidirectional permeation of water was also observed in ref. 13). These data clearly indicate that the WT channel is "water proof" in the active state, whereas the mutant channel is leaky only with the plug open. In contrast, we did not register any spontaneous ion passage during the simulations in any of the setups.

The water flux results can be explained based on an analysis of the PR diameter (Table 1 and ESI, $\dagger$ Fig. S7). PR diameters were averaged over $200 \mathrm{ps}$ time frames. The deviation of the PR diameter from the average value is characterized by the standard deviation $\sigma$ defined as:

$$
\sigma=\sqrt{\frac{1}{N-1} \sum_{i=1}^{N}\left(d_{i}-\bar{d}\right)^{2}}
$$

where $d_{i}$ is the frame-averaged distance between the c.o.m. of the respective Ile residues, $\bar{d}$ is their average distance, and $N$ is the number of frames. From Table 1, it is seen that the averaged TM2-TM10 distance of our modelled channels does not change significantly with respect to the crystal structure of the resting state for all setups. There is only a slight widening, when the

Table 1 Mean value (standard deviation) of the PR diameter in $\AA$ along the 100 ns trajectory. For comparison, the PR diameter of the resting state as inferred from the 5AWW structure is given in the last column. The increased values for MUT-open are indicated in bold

\begin{tabular}{lllllr}
\hline & WT-closed & WT-open & MUT-closed & MUT-open & 5AWW \\
\hline TM5-TM7 & $7.9(0.4)$ & $8.5(0.3)$ & $8.1(0.3)$ & $\mathbf{1 0 . 2}(\mathbf{1 . 2})$ & 7.4 \\
TM2-TM10 & $12.9(0.4)$ & $13.5(0.3)$ & $12.7(0.5)$ & $13.8(0.5)$ & 12.8
\end{tabular}


plug is open, which is somewhat larger in the mutant. The standard deviation is not significantly changed upon opening of the plug in the WT, indicating an unaltered rigidity of the PR in the TM2-TM10 direction, but is slightly increased in the mutant due to movements of TM2b for both plug positions ( $c f$. ESI, $\dagger$ Fig. S7). In the TM5-TM7 direction, we observe no significant differences between WT-closed, WT-open and MUTclosed, which all exhibit a slightly larger PR diameter than the resting state. The notable exception is the MUT-open setup, in which both the average diameter and the standard deviation are enlarged indicating a widening of the PR along the TM5TM7 direction and an increase in flexibility. Note that this increase in the PR diameter implies a decrease of the eccentricity of the ellipse defined in Fig. 1a and thus an overall widening of the PR compared to all other cases studied.
The influence of the mutation on the role of the plug in sealing the channel is even more striking when looking at probability density functions of the PR diameter (Fig. 3). For both the TM2-TM10 and the TM5-TM7 directions, the distributions of the PR diameter $d_{i}$ are relatively narrow and exhibit essentially one major maximum. Only in the case of TM5-TM7 for the MUT-open setup, the distribution is broad and even exhibits two major maxima around 10 and $12 \AA$. Generally, we observed a higher flexibility of the setups with open plug compared to those with closed plug, as is also evident from the time series of the RMSD of backbone atoms (ESI, $\uparrow$ Fig. S5; see figure caption for further details concerning the computation of RMSD). The fluctuations are the same for WT and mutant, when the plug is closed. Opening the plug increases the fluctuations in both cases, but to a significantly larger

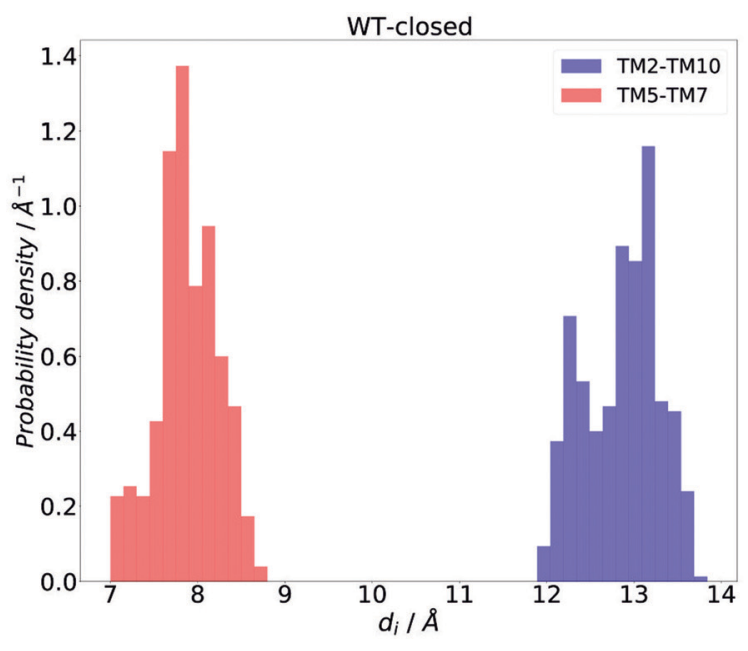

a

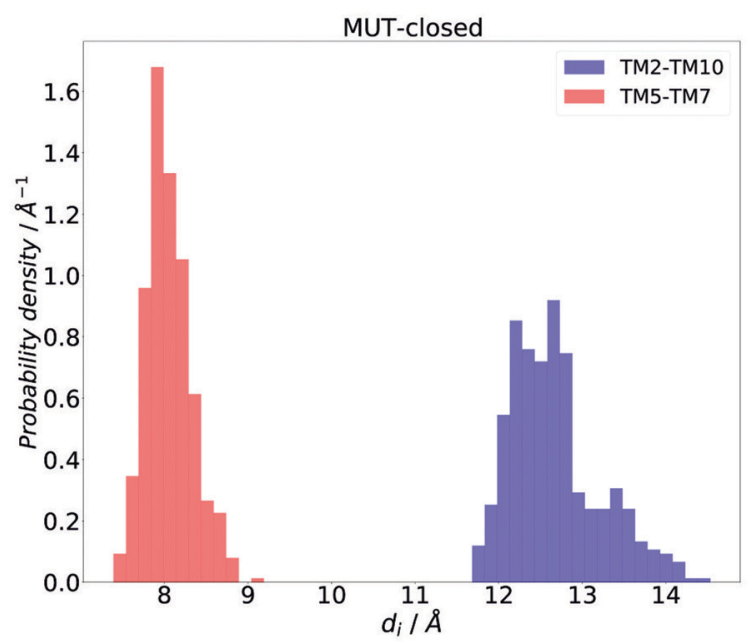

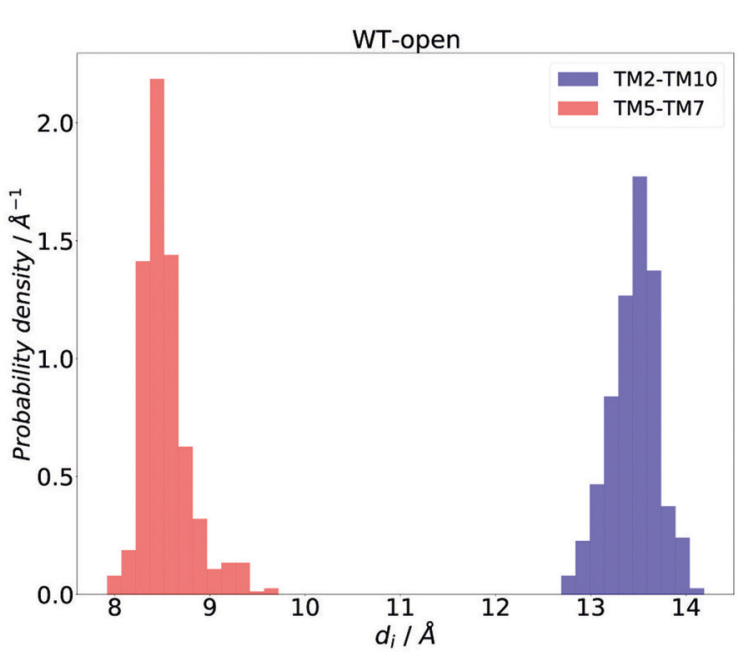

b

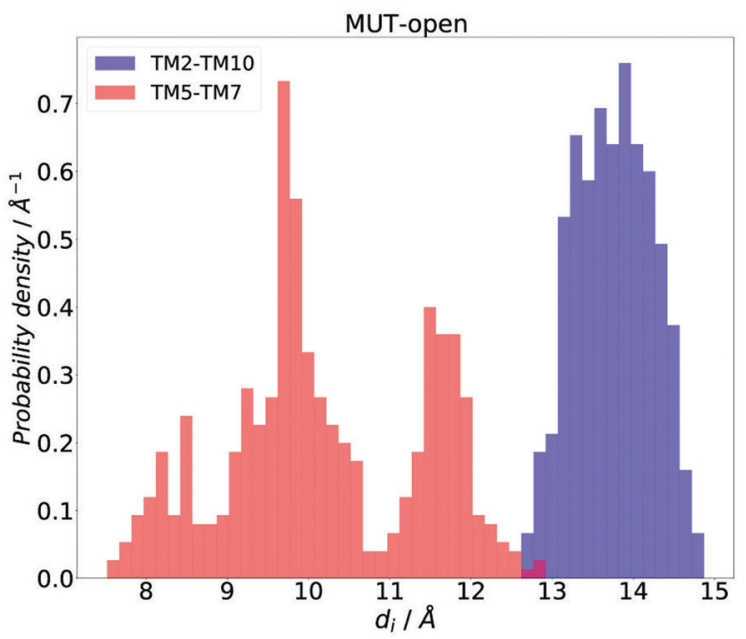

d

Fig. 3 Distributions of the PR diameter $d_{i}$ in the TM2-TM10 (blue) and TM5-TM7 (red) directions for the four setups WT-closed (a), WT-open (b), MUTclosed (c), and MUT-open (d). 
extent in the mutant. The same trend is observed for the PR diameters (ESI, $\uparrow$ Fig. S7).

The second maximum in the TM5-TM7 distance observed for MUT-open can be traced back to a sudden increase in the TM5-TM7 distance by about $2 \AA$ that is observed at $70 \mathrm{~ns}$ (ESI, $\dagger$ Fig. S7b). A detailed analysis of this conformational switch reveals that the short helices TM7 and TM2b undergo a translational shift, while the long helices TM10 and TM5 tilt and rotate. The large-amplitude jump of the TM5-TM7 distance of the PR at $70 \mathrm{~ns}$ starts with a tilt and rotation of TM5 that can be seen by comparing the position of Ile 188 at 65 and 72 ns (ESI, $\dagger$ Fig. S8). Interestingly, TM5 rotates "away" from the TM10 helix, which harbors the mutated residue 403. Very likely, the TM5-TM10 interaction ( $c f$. Fig. 1a) is weakened due to a perturbation of hydrophobic interactions by replacing Ile 403 with the polar Asn. This effect points to the importance of hydrophobic interactions within the PR. Furthermore, the tilt of TM5, being accompanied by a shift of TM7, progresses in time during the MD simulation, increasing the separation between the PR residues Ile 188 and Ile 275. At the end of the trajectory, the TM5-TM7 distance reaches the magnitude of the TM2-TM10 distance (ESI, $\dagger$ Fig. S7b), implying that the ellipse (cf. Fig. 1a) has essentially become a circle. Taking into account, that the major part of the 284 water molecules passed the channel after 70 ns (see ESI, $\dagger$ Fig. S9), we conclude that the eccentricity of the PR with the TM5-TM7 distance being smaller than the TM2-TM10 distance provides the major barrier to the water flux. The fluctuations of the TM2b and TM10 positions do not contribute much. Also, TM5 is very large and has not enough space to shift; it can only rotate or bend. Thus, we suggest that opening of the channel must involve, first of all, an enlargement of the TM5-TM7 distance and, hence, a movement of TM7.

The time series of the total number of water molecule crossing the PR in the MUT-open setup (ESI, $\dagger$ Fig. S9) shows that after the conformational switch, the system reaches a new equilibrium state (see also the RMSD time series in ESI, $\dagger$ Fig. S5) with a constant (bidirectional) water translocation of 6 molecules per ns.

For the open conformations of both WT and the mutant, the plug is moving up and sideward, reducing the distance to the PR and TM4. However, it does not reach the initial configuration it had in the closed conformation, probably, due to significant deformations as a result of pulling by SMD (ESI, $\dagger$ Fig. S10).

The above data lead us to a surprising observation: with an open LG and plug, the WT channel still manages to keep the PR quite tight as in the resting state, so that only about 10 water molecules can cross the channel in 100 ns. Moreover, the presence of the mutation in the PR does not break the barrier either, provided the plug is closed. Only the mutant with the open plug allows for significant water flux. Our results demonstrate that the plug and the LG are to a certain extent independent of each other: Opening of the LG can proceed without significant changes in the plug position. This conclusion is in agreement with ref. 10 .

\subsection{Free energy barrier of ion passage}

Since no ions were detected to cross the channel during an unbiased 100 ns dynamics (see Section 3.1), we performed SMD and US simulations to determine the forces and the free energy barrier for both $\mathrm{Cl}^{-}$anions and $\mathrm{K}^{+}$cations (see Methods for details). The forces exerted on the ions along the channel's axis obtained from SMD simulations are shown in Fig. 4. (The time dependence of the ion position along the $z$-axis is given in ESI, $\dagger$ Fig. S11). Interestingly, the difference between maximal forces, corresponding to a position of the $\mathrm{Cl}^{-}$ion in the PR area, is in the same order of magnitude as the error bars, implying that the forces are essentially the same for the channel with open and closed plug. This result provides evidence that the PR alone can act as an efficient barrier for the ion flux.

The difference in the peak positions around $z=0 \AA$ in Fig. 4 is caused by the different paths taken by the ions. The paths are determined by both the spatial distribution of amino acid residues, which act as obstacles, and the distribution of electric fields produced by charged and polar residues of the channel. Moreover, the position of the plug close to the PR (in the closed state) can also influence the ion's trajectory by providing a spatial obstacle and inducing electrostatic interactions with the ion.

The extracted forces can be used for calculations of the free energy with the help of the Jarzynski equality. ${ }^{36}$ According to this equation, the free energy difference $\Delta F$ between two states can be calculated from the average work $W$ in a nonequilibrium process:

$$
\mathrm{e}^{-\beta \Delta F}=\left\langle\mathrm{e}^{-\beta W}\right\rangle
$$

where $\beta=1 /\left(k_{\mathrm{B}} T\right)$. In the stiff-spring approximation, ${ }^{37}$ when the reaction coordinate closely follows the constraint center, the work $W_{1 \rightarrow 2}$ performed along the path of the SMD trajectory while driving the system from state 1 to state 2 with constant velocity can be connected to the free energy difference between initial and final states:

$$
\Delta F=-\beta^{-1} \ln \left\{\left\langle\exp \left\{-\beta W_{1 \rightarrow 2}\right\}\right\rangle\right\}
$$

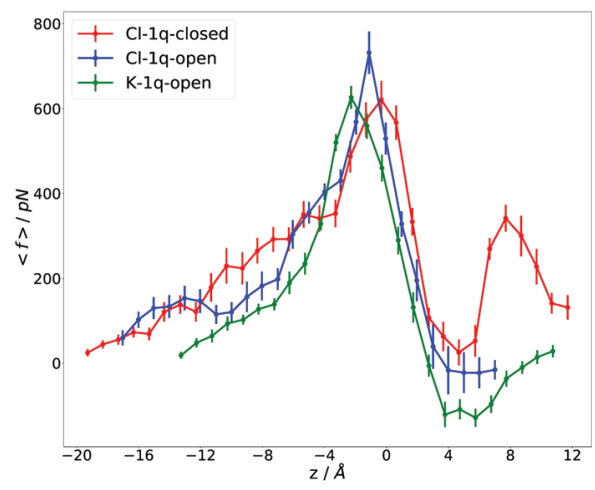

Fig. 4 Average force $f$ acting on the ion as a function of the ion's position along the collective variable - the relative $z$-coordinate defined in Fig. 2 for the setups $\mathrm{Cl}-1 \mathrm{q}$-open (blue), $\mathrm{Cl}-1 \mathrm{q}$-closed (red), and $\mathrm{K}$-1q-open (green). 


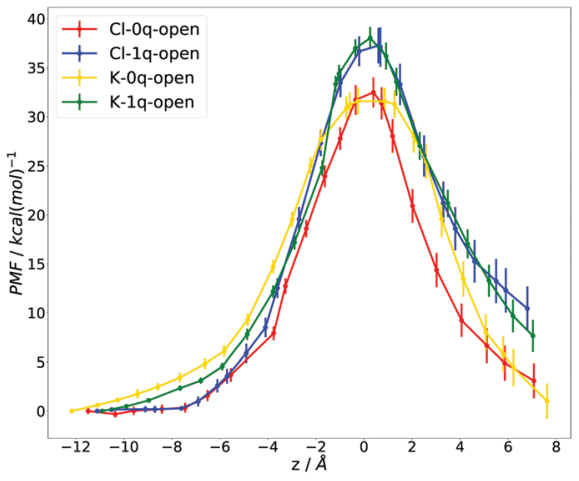

Fig. 5 Potential of mean force (PMF) as a function of the ion's position along the collective variable - the relative $z$-coordinate defined in Fig. 2 for the setups $\mathrm{Cl}-0 \mathrm{q}$-open (red), $\mathrm{Cl}-1 \mathrm{q}$-open (blue), $\mathrm{K}$-Oq-open (orange), and $\mathrm{K}-1 \mathrm{q}$-open (green).

Here, the average is taken over the ensemble of trajectories with different starting velocities.

We calculated the free energy difference between the initial state, corresponding to the $\mathrm{Cl}^{-}$or $\mathrm{K}^{+}$ion located in the cytoplasmic part of the channel $(z=-11 \AA)$, and the final state, corresponding to the respective ion being located in the pore ring system $(z=0 \AA)$, where the forces are maximal. Averaging over 30 trajectories yields $33 \pm 3$ and $29 \pm 3 \mathrm{kcal} \mathrm{mol}^{-1}$ for $\mathrm{Cl}^{-}$ and $\mathrm{K}^{+}$ions, respectively, with the plug open, confirming that the differences in barriers are within the error limits. It may be noticed that there is a second maximum in the $f(z)$ curve for Cl1q-closed, which is due to interaction of the ion with the plug. Thus, while the plug in its closed conformation provides a barrier against ion flux, this barrier is not crucial in the WT, since ion permeation is already efficiently prevented by the PR.

The results of the US calculations, representing the dependence of the PMF on the relative distance between ion and PR, are shown in Fig. 5 (for details, see Methods; probability density functions of the US simulations are given in ESI, $\dagger$ Fig. S6; a description of the error calculations - based on ref. 38 - is presented in ESI, $\uparrow$ Fig. S12). We can compare the free energy difference for the setups Cl-1q-open and K-1q-open with the results of the SMD simulations. The PMF difference between the states corresponding to $z=0 \AA$ and $z=-11 \AA$ equals $38 \pm$ $2 \mathrm{kcal} \mathrm{mol}^{-1}$ for both ions, which is in quite good agreement with the SMD results. The remaining discrepancy is likely due to the limited sampling in SMD. The PMF profiles show that the free energy barriers have the same magnitude for $\mathrm{Cl}^{-}$and $\mathrm{K}^{+}$in the setups with the same charge state of Asp 410. For both ions, the barrier is decreased by about $5 \mathrm{kcal} \mathrm{mol}^{-1}$, when Asp 410 is uncharged.

To better understand the factors contributing to the PMF barrier, we analyzed the enthalpic part of the free energy difference, $\Delta H$, by comparing electrostatic and van der Waals interactions in the two states corresponding to $z=-1 \AA$ and $z=-11 \AA$ A. The following components of $\Delta H$ were distinguished: the coupling between protein (P) and ion ( $\mathrm{I}$; the $\mathrm{Cl}^{-}$or $\mathrm{K}^{+}$ion), $\Delta H_{\mathrm{PI}}$, between water $(\mathrm{W})$ and ion, $\Delta H_{\mathrm{WI}}$, between water molecules, $\Delta H_{\mathrm{WW}}$, as well as between protein and water, $\Delta H_{\mathrm{PW}}$.
Table 2 Mean interaction enthalpy differences (standard errors) in $\mathrm{kcal} \mathrm{mol}^{-1}$ between the two trajectories Traj1 and Traj2 decomposed as described in the text

\begin{tabular}{lllll}
\hline Setup & $\Delta H_{\mathrm{PI}}$ & $\Delta H_{\mathrm{WI}}$ & $\Delta H_{\mathrm{WW}}$ & $\Delta H_{\mathrm{PW}}$ \\
\hline Cl-0q-open & $-53.7(1.8)$ & $60.6(2.1)$ & $-106.6(36.7)$ & $85.6(27.2)$ \\
Cl-1q-open & $-54.6(1.4)$ & $64.9(1.8)$ & $-101.4(32.4)$ & $156.6(34.8)$ \\
K-0q-open & $30.3(3.7)$ & $-15.7(3.4)$ & $216.9(38.3)$ & $-233.5(29.6)$ \\
K-1q-open & $31.3(2.8)$ & $-18.9(3.0)$ & $80.9(38.8)$ & $-121.8(26.8)$
\end{tabular}

The interactions were computed from 60 ns trajectories (see Methods) corresponding to $z=-11 \AA$ (Traj1) and $z=-1 \AA$ (Traj2). (The fluctuations of interaction energies corresponding to the window with $z=0 \AA$ were too high to provide reliable results in calculations of enthalpy differences.) The mean differences between averaged interaction energies were calculated by subtracting the value obtained for Traj 1 from that for Traj2. The computed differences for every type of interaction were analyzed with the independent-samples $t$-test (Student's $t$-test) performed with the SPSS Statistics package ${ }^{39}$ (for details, see ESI, $\dagger$ Section $\mathrm{T} 1$ and ref. 40). The test demonstrated statistically significant differences between the means of the interaction energies for all cases. The mean differences and their standard errors are given in Table 2.

Analysis of the data in Table 2 reveals that interaction energy differences of the same type have different signs for $\mathrm{K}^{+}$and $\mathrm{Cl}^{-}$. While PW and WI interactions make a positive enthalpic contribution to the free energy barrier for the $\mathrm{Cl}^{-}$ion, WW and PI interaction changes are positive in the case of $\mathrm{K}^{+}$. The charge state of Asp 410 has a negligible influence on $\Delta H_{\mathrm{PI}}$ and $\Delta H_{\mathrm{WI}}$. Therefore, we can write for the change in $\Delta H$ due to charging of the Asp side chain:

$$
\begin{gathered}
\Delta H(1 \mathrm{q})-\Delta H(0 \mathrm{q}) \approx \Delta H_{\mathrm{WW}}(1 \mathrm{q})-\Delta H_{\mathrm{WW}}(0 \mathrm{q})+\Delta H_{\mathrm{PW}}(1 \mathrm{q}) \\
-\Delta H_{\mathrm{PW}}(0 \mathrm{q})
\end{gathered}
$$

There is a significant positive contribution to the difference in $\Delta H$ between charged and uncharged Asp 410 due to proteinwater interactions of $71 \pm 44 \mathrm{kcal} \mathrm{mol}^{-1}$ for $\mathrm{Cl}^{-}$and $112 \pm$ $40 \mathrm{kcal} \mathrm{mol}^{-1}$ for $\mathrm{K}^{+}$, which - in view of the standard errors - is essentially the same for both ions and likely due to the coupling of Asp 410 to water molecules inside the channel. Interestingly, there is a second contribution from water-water interactions, which is $6 \pm 49 \mathrm{kcal} \mathrm{mol}^{-1}$ for $\mathrm{Cl}^{-}$, but $-136 \pm 55 \mathrm{kcal} \mathrm{mol}^{-1}$ for $\mathrm{K}^{+}$; this is different even taking into account the standard errors. The disparity between the two types of ions is likely related to their influence on the water network in the channel, as different signs of the charge enforce different orientations of the water molecules (see snapshots in ESI, $\dagger$ Fig. S13). Thus, the charge state of Asp 410 influences the enthalpic barriers for the variously charged ions in a different way as one might have expected, but the effect is not due to direct Asp-ion interactions, but rather indirect due to WW and PW interactions. However, the differences in the enthalpic barrier are efficiently counterbalanced by entropy changes, as is obvious from the free energy barriers, which are not only the same for both ions, but also 
shifted by the same amount of only $5 \mathrm{kcal} \mathrm{mol}^{-1}$ due to a change in the charge state of Asp 410 (Fig. 5).

To see what determines the difference between $\mathrm{Cl}^{-}$and $\mathrm{K}^{+}$, we have to consider all contributions to $\Delta H$, i.e.:

$$
\begin{gathered}
\Delta H(\mathrm{Cl})-\Delta H(\mathrm{~K})=\Delta H_{\mathrm{PI}}(\mathrm{Cl})-\Delta H_{\mathrm{PI}}(\mathrm{K})+\Delta H_{\mathrm{WI}}(\mathrm{Cl})-\Delta H_{\mathrm{WI}}(\mathrm{K}) \\
+\Delta H_{\mathrm{WW}}(\mathrm{Cl})-\Delta H_{\mathrm{WW}}(\mathrm{K})+\Delta H_{\mathrm{PW}}(\mathrm{Cl})-\Delta H_{\mathrm{PW}}(\mathrm{K})
\end{gathered}
$$

In the case of uncharged Asp 410, we have (Table 2):

$$
\begin{gathered}
\Delta H_{\mathrm{PI}}(\mathrm{Cl})-\Delta H_{\mathrm{PI}}(\mathrm{K}) \approx-\left(\Delta H_{\mathrm{WI}}(\mathrm{Cl})-\Delta H_{\mathrm{WI}}(\mathrm{K})\right) \\
\Delta H_{\mathrm{WW}}(\mathrm{Cl})-\Delta H_{\mathrm{WW}}(\mathrm{K}) \approx-\left(\Delta H_{\mathrm{PW}}(\mathrm{Cl})-\Delta H_{\mathrm{PW}}(\mathrm{K})\right)
\end{gathered}
$$

Thus, there is a compensating trend, indicating correlations between PI and WI as well as WW and PW interactions, resulting in a negligible value of $\Delta H(\mathrm{Cl})-\Delta H(\mathrm{~K})$. The situation is different for charged Asp 410: the WW and PW interactions do not compensate each other, and we obtain $\Delta H(\mathrm{Cl})-\Delta H(\mathrm{~K})=$ $94 \pm 67 \mathrm{kcal} \mathrm{mol}^{-1}$. Again, this difference is entirely counterbalanced by entropy changes.

Thus, to summarize these unexpected results: The free energy barrier to ion passage through the translocon is the same for oppositely charged (monovalent) ions and only marginally affected by the (negative) charge of a nearby amino acid side chain in the channel. The enthalpic contribution to the barrier is dominated by changes in water-water and proteinwater interactions.

\section{Discussion}

The results of our studies demonstrate, first of all, that the translocon in the active state, i.e. with a SS occupying the LG, provides a tight barrier for both water and small ions. The seal is provided by hydrophobic interactions between TM7, plug and PR residues, which we refer to as the hydrophobic core of $\mathrm{SecY}$, and which is also relevant for sealing the channel in the resting state (Fig. 6). These interactions, presumably, stimulate folding of SecYEG into a compact and tight conformation: The plug is very close to the PR, the PR residues are close to each other, and opening of the LG by virtue of inserting the SS does not break the hydrophobic interactions. The same type of hydrophobic core is present in other organisms (ESI, $\dagger$ Fig. S14).

Secondly, leakage of the translocon is triggered by a high mobility of the TM7 helix resulting from weakened interactions in the hydrophobic core. If some of these interactions are still present, like in the conformations WT-open or MUT-closed, the channel is sealed. In the former case, interactions between Ile residues of the PR help to keep the barrier, while in the second case the plug prevents fluctuations of TM7. The importance of the interactions between plug, TM7 and TM2b was also discussed in ref. 6, where an analysis of the structure 2YXR, corresponding to plug-deleted SecY, demonstrated that the newly formed plug is moved away from the PR compared to the location of the plug in the WT structure. Moreover, the new plug lost almost all interactions with TM2b and TM7.

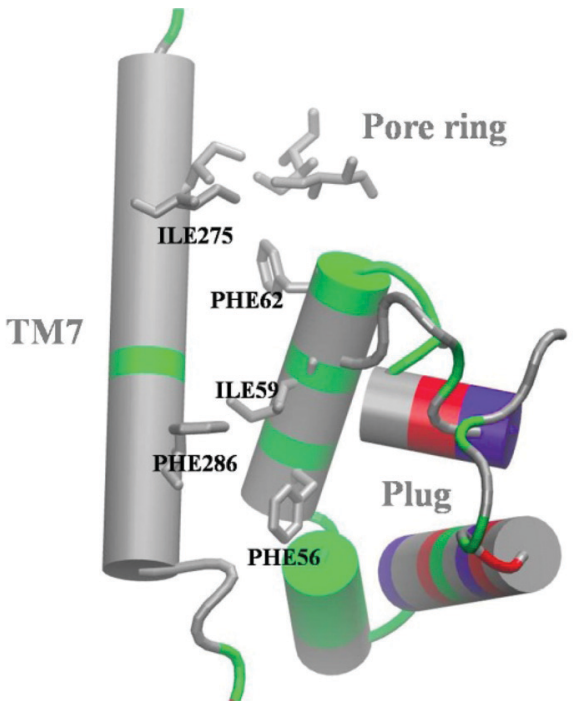

Fig. 6 Hydrophobic core interactions in the resting channel (based on crystal structure 5AWW). Color code for amino acid types: grey, hydrophobic; green, polar; blue, positively charged; red, negatively charged (the latter two in the standard protonation state). Hydrophobic residues in close proximity are explicitly shown.

Taking into account that the plug-deleted channels are leaky, we can speculate that the absence of the plug may prevent formation of the compact structure, which results in weakening of the hydrophobic interactions and, presumably, loosening of TM7. Similar considerations apply to PR mutants: substitution of Ile residues in the PR with polar ones weakens the interactions among the PR residues and, at the same time, those with the plug, which may lead to a channel with widened PR and shifted plug. Confirmation of this hypothesis requires analysis of high-resolution structures of mutants, which are not yet available.

Our results also suggest a possible interpretation of electrophysiological experiments, ${ }^{8}$ where the channel, activated by inserting a SS, exhibited a voltage sensitivity of ion permeation. We speculate that TM7 might be a voltage sensor due to the net dipole moment of the $\alpha$-helix backbone. The position of TM7 critically determines the minimal width of the pore ring and even a $2 \AA$ shift can significantly affect the permeability of the channel as shown for water in the present work. The TM7 helix does not contain any charged residues and is composed, mainly, of hydrophobic amino acids (see Fig. 6). The helix dipole amounts to $55 \mathrm{D}$ (ESI, $\dagger$ Fig. S15). Application of an electric field could result in a tilt or rotation of the helix and, in this way, affect the tightness of the PR/plug region similar to what is observed in the present work for the MUT-open setup. Further MD simulations with applied voltage will be necessary to clarify this issue.

The enthalpic part of the barrier for ion transport through SecYEG is determined by different electrostatic couplings, depending on the sign of the ion's charge, whereby changes in protein-water and water-water interactions are important. The water interior of the translocon was already subject to 
investigations. ${ }^{41,42}$ It was shown that water in the channel behaves differently from bulk water exhibiting anomalous diffusion, retarded rotational dynamics and alignment of the dipoles along the channel's axis. These data - taken together with our results - show that water plays a critical role in translocon function. A further detailed analysis of the waternetwork in the channel is required to understand its contribution to electrostatic couplings and free energy barriers.

The phenomenon of enthalpy-entropy compensation has been studied experimentally and theoretically mainly in the context of protein-ligand binding (for recent reviews, see ref. 43 and 44). Here, we observe - to the best of our knowledge for the first time - a similar effect in the context of ion permeation through a membrane channel. Clearly, oppositely charged ions will interact differently with a given channel, so that the existence of an enthalpic barrier to ion permeation is not warranted for all kinds of ions. Our data clearly show the differences in $\Delta H$ for $\mathrm{K}^{+}$and $\mathrm{Cl}^{-}$. It is reasonable, then, that compensating entropy changes come to the rescue of the channel's impermeability. Thus, the sealing of protein channels and nanopores against ion leakage may be considered as a new field, in which enthalpy-entropy compensation is relevant.

In this respect, it is interesting to compare our results with data from Baron et al., ${ }^{45}$ who computed the free energy, enthalpy and entropy change for binding of a charged ligand to a nonpolar cavity based on US. It was found that the unfavorable $\Delta G$ for ligand binding is caused by a large positive $\Delta H$ due to loss of water-water interactions that is only partly compensated by the entropy term. This trend does not depend on the sign of the ligand's charge. In contrast, we observe a dependence of the sign of $\Delta H_{\mathrm{Ww}}$ on the sign of the ion's charge. The difference is likely due to the presence of charged and polar amino acid residues in the translocon's "walls". Baron et $a l^{45}$ also observe a switch of $\Delta H_{\mathrm{WW}}$ from positive to negative, when they place an appropriate charge in the cavity wall. In our case, however, there is remarkably perfect enthalpy-entropy compensation, so that the translocon is not able to distinguish between $\mathrm{K}^{+}$and $\mathrm{Cl}^{-}$regarding the permeation barrier. It should be noted that this indifference for the ion type may be removed by a mutation-induced weakening of interactions in the hydrophobic core or by an applied transmembrane potential. Hence, our data are not necessarily in contradiction to experiments, in which anion selectivity of SecYEG was observed. ${ }^{8}$ As mentioned above, the effects of a membrane potential remain to be investigated.

Charged amino acid residues are located in spatial proximity to the PR in the translocon of many organisms (see ESI, $\dagger$ Table S1). We wondered whether such a charged group may influence the barrier to ion penetration or contribute to ion selectivity. Remarkably, a negative charge on Asp 410 in T. thermophilus increases the free energy barrier for both ion types by the same amount, while the barrier remains sufficiently large to block ion permeation in the absence of the charge. Thus, the role of a charged group close to the $\mathrm{PR}$ remains elusive. One may speculate, though, that the $5 \mathrm{kcal} \mathrm{mol}^{-1}$ difference in the free energy barrier due to
Asp 410 becomes important, when the translocon's structure is perturbed as in mutants or under a membrane potential.

The large free energy barrier for ions raises an important question regarding the mechanism of protein translocation: how can a peptide bearing a charged side chain be transported through the channel? As we shall show in a forthcoming publication, the case of a charge on a peptide is significantly different from that of an ion.

\section{Conclusions}

The SecYEG channel in the active state, i.e., with a SS helix occupying the LG, provides a strong barrier to water and ion flux. Although the PR is slightly widened in the WT translocon compared to the resting state, hydrophobic interactions between the plug, TM7 and the PR - constituting a hydrophobic core - are still able to keep the pore tight. The PR alone is effectively sealing the WT translocon. Perturbing the hydrophobic core, e.g., by mutations of PR residues, can lead to water leakage, if a significant part of the hydrophobic interactions is disrupted, which depends on the position of the plug. Thus, the role of the plug in sealing the channel is not unequivocal, but it seems to be of minor importance for the WT. The plug does not play a role in sealing the channel against ion permeation either, while the PR provides a significant free energy barrier for $\mathrm{Cl}^{-}$ and $\mathrm{K}^{+}$. Due to remarkable enthalpy-entropy compensation, this barrier is the same for both types of ions. The negative charge on a nearby aspartate does not contribute significantly to the barrier. Important open questions that remain to be tackled in future work are related to the translocation of peptides with charged groups and the influence of a membrane potential on the free energy barrier.

\section{Conflicts of interest}

There are no conflicts to declare.

\section{Acknowledgements}

We would like to thank Ferdinand Horvath (Institute for Theoretical Physics, Johannes Kepler University Linz) as well as Dr Mirjam Zimmermann, Dr Denis Knyazev, and Prof. Dr Peter Pohl (Institute for Biophysics, Johannes Kepler University Linz) for collaboration. Very special thanks to Prof. Dr ErnstWalter Knapp (Free University of Berlin) and his group (Dr Jovan Draglj, Dr Nadia Elgobashi-Meinhardt, Dr Milan Hodoscek) for help in the early stages of the project. This work was supported by the Doctoral Program "Nano-Analytics of Cellular Systems (NanoCell)" of the Austrian Science Fund (FWF) (grant W 1250), project P 33154-B funded by the FWF in conjunction with the federal state of Upper Austria, and the Linz Institute of Technology (grant LIT-2019-8-SEE-120). 


\section{Notes and references}

1 J. Oswald, R. Njenga, A. Natriashvili, P. Sarmah and H. G. Koch, Front. Mol. Biosci., 2021, 8, 664241.

2 T. A. Rapoport, L. Li and E. Park, Annu. Rev. Cell Dev. Biol., 2017, 33, 369-390.

3 D. G. Knyazev, R. Kuttner, M. Zimmermann, E. Sobakinskaya and P. Pohl, J. Membr. Biol., 2018, 251, 329-343.

4 S. M. Saparov, K. Erlandson, K. Cannon, J. Schaletzky, S. Schulman, T. A. Rapoport and P. Pohl, Mol. Cell, 2007, 26, 501-509.

5 E. Park and T. A. Rapoport, Nature, 2011, 473, 239-242.

6 W. Li, S. Schulman, D. Boyd, K. Erlandson, J. Beckwith and T. A. Rapoport, Mol. Cell, 2007, 26, 511-521.

7 J. Gumbart and K. Schulten, J. Gen. Physiol., 2008, 132, 709-719.

8 D. G. Knyazev, L. Winter, B. W. Bauer, C. Siligan and P. Pohl, J. Biol. Chem., 2014, 289, 24611-24616.

9 B. van den Berg, W. M. Clemons Jr, I. Collinson, Y. Modis, E. Hartmann, S. C. Harrison and T. A. Rapoport, Nature, 2004, 427, 36-44.

10 P. Tian and I. Andricioaei, Biophys. J., 2006, 90, 2718-2730.

11 J. Gumbart and K. Schulten, Biochemistry, 2007, 46, 11147-11157.

12 S. J. Sun, S. S. Wang, Z. F. Tong, X. D. Yao and J. Gao, RSC Adv., 2019, 9, 14876-14883.

13 J. Gumbart and K. Schulten, Biophys. J., 2006, 90, 2356-2367.

14 B. Zhang and T. F. Miller 3rd, Proc. Natl. Acad. Sci. U. S. A., 2010, 107, 5399-5404.

15 L. Li, E. Park, J. Ling, J. Ingram, H. Ploegh and T. A. Rapoport, Nature, 2016, 531, 395-399.

16 Y. Tanaka, Y. Sugano, M. Takemoto, T. Mori, A. Furukawa, T. Kusakizako, K. Kumazaki, A. Kashima, R. Ishitani, Y. Sugita, O. Nureki and T. Tsukazaki, Cell Rep., 2015, 13, 1561-1568.

17 J. C. Phillips, D. J. Hardy, J. D. C. Maia, J. E. Stone, J. V. Ribeiro, R. C. Bernardi, R. Buch, G. Fiorin, J. Henin, W. Jiang, R. McGreevy, M. C. R. Melo, B. K. Radak, R. D. Skeel, A. Singharoy, Y. Wang, B. Roux, A. Aksimentiev, Z. Luthey-Schulten, L. V. Kale, K. Schulten, C. Chipot and E. Tajkhorshid, J. Chem. Phys., 2020, 153, 044130.

18 S. Jo, T. Kim, V. G. Iyer and W. Im, J. Comput. Chem., 2008, 29, 1859-1865.

19 B. R. Brooks, C. L. Brooks 3rd, A. D. Mackerell Jr, L. Nilsson, R. J. Petrella, B. Roux, Y. Won, G. Archontis, C. Bartels, S. Boresch, A. Caflisch, L. Caves, Q. Cui, A. R. Dinner, M. Feig, S. Fischer, J. Gao, M. Hodoscek, W. Im, K. Kuczera, T. Lazaridis, J. Ma, V. Ovchinnikov, E. Paci, R. W. Pastor, C. B. Post, J. Z. Pu, M. Schaefer, B. Tidor,
R. M. Venable, H. L. Woodcock, X. Wu, W. Yang, D. M. York and M. Karplus, J. Comput. Chem., 2009, 30, 1545-1614.

20 J. Lee, X. Cheng, J. M. Swails, M. S. Yeom, P. K. Eastman, J. A. Lemkul, S. Wei, J. Buckner, J. C. Jeong, Y. Qi, S. Jo, V. S. Pande, D. A. Case, C. L. Brooks 3rd, A. D. MacKerell Jr, J. B. Klauda and W. Im, J. Chem. Theory Comput., 2016, 12, 405-413.

21 J. R. Willdigg and J. D. Helmann, Front. Mol. Biosci., 2021, 8, 634438.

22 H. Lu and K. Schulten, Proteins, 1999, 35, 453-463.

23 A. D. Mackerell Jr, J. Comput. Chem., 2004, 25, 1584-1604.

24 X. Zhu, P. E. Lopes and A. D. Mackerell Jr, Wiley Interdiscip. Rev.: Comput. Mol. Sci., 2012, 2, 167-185.

25 T. Schlick, Molecular Modeling and Simulation: An Interdisciplinary Guide, Springer, New York, 2010.

26 G. J. Martyna, D. J. Tobias and M. L. Klein, J. Chem. Phys., 1994, 101, 4177-4189.

27 S. E. Feller, Y. H. Zhang, R. W. Pastor and B. R. Brooks, J. Chem. Phys., 1995, 103, 4613-4621.

28 W. Humphrey, A. Dalke and K. Schulten, J. Mol. Graphics, 1996, 14(33-38), 27-38.

29 https:/www.ks.uiuc.edu/Training/Tutorials/.

30 G. M. Torrie and J. P. Valleau, Chem. Phys. Lett., 1974, 28, 578-581.

31 G. M. Torrie and J. P. Valleau, J. Comput. Phys., 1977, 23, 187-199.

32 J. Kästner, Wiley Interdiscip. Rev.: Comput. Mol. Sci., 2011, 1, 932-942.

33 S. Kumar, D. Bouzida, R. H. Swendsen, P. A. Kollman and J. M. Rosenberg, J. Comput. Chem., 1992, 13, 1011-1021.

34 http://membrane.urmc.rochester.edu/?page_id=126.

35 https://www.ks.uiuc.edu/Research/namd/2.9/ug/node74. html.

36 C. Jarzynski, Phys. Rev. Lett., 1997, 78, 2690-2693.

37 S. Park, F. Khalili-Araghi, E. Tajkhorshid and K. Schulten, J. Chem. Phys., 2003, 119, 3559-3566.

38 F. Zhu and G. Hummer, J. Comput. Chem., 2012, 33, 453-465.

39 https://www.ibm.com.

40 https://statistics.laerd.com.

41 S. Capponi, M. Heyden, A. N. Bondar, D. J. Tobias and S. H. White, Proc. Natl. Acad. Sci. U. S. A., 2015, 112, 9016-9021.

42 S. Capponi, S. H. White, D. J. Tobias and M. Heyden, J. Phys. Chem. B, 2019, 123, 480-486.

43 J. M. Fox, M. X. Zhao, M. J. Fink, K. Kang and G. M. Whitesides, Annu. Rev. Biophys., 2018, 47, 223-250.

44 F. Peccati and G. Jimenez-Oses, ACS Omega, 2021, 6, 11122-11130.

45 R. Baron, P. Setny and J. A. McCammon, J. Am. Chem. Soc., 2010, 132, 12091-12097. 